B. Chakraborty, W. LÜ

\title{
ON THE VALUE DISTRIBUTION OF A DIFFERENTIAL MONOMIAL AND SOME NORMALITY CRITERIA
}

\footnotetext{
B. Chakraborty, W. Lü.On the value distribution of a differential monomial and some normality criteria, Mat. Stud. 56 (2021), 55-60.

The aim of this paper is to study the zero distribution of the differential polynomial

$$
a f^{q_{0}}\left(f^{\prime}\right)^{q_{1}} \ldots\left(f^{(k)}\right)^{q_{k}}-\varphi
$$

where $f$ is a transcendental meromorphic function and $a=a(z)(\not \equiv 0, \infty)$ and $\varphi(\not \equiv 0, \infty)$ are small functions of $f$. Moreover, using this value distribution result, we prove the following normality criterion for family of analytic functions:

Let $\mathscr{F}$ be a family of analytic functions on a domain $D$ and let $k \geq 1, q_{0} \geq 2, q_{i} \geq 0$ $(i=1,2, \ldots, k-1), q_{k} \geq 1$ be positive integers. If for each $f \in \mathscr{F}: \bar{i}$. $f$ has only zeros of multiplicity at least $k$, ii.

then $\mathscr{F}$ is normal on domain D.

$$
f^{q_{0}}\left(f^{\prime}\right)^{q_{1}} \ldots\left(f^{(k)}\right)^{q_{k}} \neq 1
$$
}

1. Introduction. The topic of this article has its origin in Hayman's ([3]) result that if $f$ is a transcendental meromorphic function and $n \geq 3$, then $f^{n} f^{\prime}$ assumes all finite values except possibly zero infinitely often.

Later this result was complemented by E. Mues ([8]) (for $n=2$ ) and H. Y. Chen and M. L. Fang ([1]) (for $n=1$ ). Using Bloch's principle and Mues's result ([8]), in 1989, X. C. Pang ([9]) gave an analogous theorem for meromorphic functions in the unit disc (or bounded domain) in terms of normality of a family of meromorphic functions as follows:

Theorem A. ([9]) Let $\mathscr{F}$ be a family of meromorphic function on a domain $D$. If each $f \in \mathscr{F}$ satisfies $f^{2} f^{\prime} \neq 1$, then $\mathscr{F}$ is normal on domain $D$.

The result of Mues was qualitative result. In 1992, Q. Zhang ([16]) gave the quantitative version of Mues's result as follows:

Theorem B. For a transcendental meromorphic function $f$, the following inequality holds

$$
T(r, f) \leq 6 N\left(r, \frac{1}{f^{2} f^{\prime}-1}\right)+S(r, f) .
$$

In this direction, X. Huang and Y. Gu ([4]) further extended the Zhang's result ([16]) by replacing $f^{\prime}$ by $f^{(k)},(k \in \mathbb{N})$.

Theorem C. ([4]) Let $f$ be a transcendental meromorphic function and $k$ be a positive integer. Then

$$
T(r, f) \leq 6 N\left(r, \frac{1}{f^{2} f^{(k)}-1}\right)+S(r, f) .
$$

2010 Mathematics Subject Classification: 30D20, 30D30, 30D35, 30 D45.

Keywords: value distribution theory; transcendental meromorphic function; differential monomials; normal family.

doi:10.30970/ms.56.1.55-60

(C) B. Chakraborty, W. Lü, 2021 
Moreover, in the same paper, C. X. Huang and Y. Gu ([4]) proved the following normality criterion for family of meromorphic functions:

Theorem D. ([4]) Let $\mathscr{F}$ be a family of meromorphic functions on a domain $D$ and let $k$ be a positive integer. If for each $f \in \mathscr{F}, f$ has only zeros of multiplicity at least $k$ and $f^{2} f^{(k)} \neq 1$, then $\mathscr{F}$ is normal on domain $D$.

To study the value distribution of a differential polynomial in more general settings, in 2003, I. Lahiri and S. Dewan ([6]) proved the following theorem:

Theorem E. Let $f$ be a transcendental meromorphic function and $\alpha=\alpha(z)(\not \equiv 0, \infty)$ be a small function of $f$. If $\psi=\alpha(f)^{n}\left(f^{(k)}\right)^{p}$, where $n(\geq 0) p(\geq 1), k(\geq 1)$ are integers, then for any small function $a=a(z)(\not \equiv 0, \infty)$ of $\psi$,

$$
(p+n) T(r, f) \leq \bar{N}(r, \infty ; f)+\bar{N}(r, 0 ; f)+p N_{k}(r, 0 ; f)+\bar{N}(r, a ; \psi)+S(r, f),
$$

where $N_{k}(r, 0 ; f)$ the counting function of zeros of $f$, where a zero of $f$ with multiplicity $q$ is counted $q$ times if $q \leq k$, and is counted $k$ times if $q>k$.

In this direction, a lot of investigations were made (e.g., ([12]), ([13]), ([14]), ([15])). Moreover, one can go through the Steinmetz' book, Nevanlinna theory, normal families, and algebraic differential equations ([11]) for the generalizations the Hayman result (Chapter 3, Section 3.2.).

Moreover, Theorem 4.12 of the same book ([11]) gave the following normality criterion:

Theorem F. ([11]) Let $k \geq 1$ and $n \geq 1$ be integers, and $\mathscr{F}$ be a family of analytic functions $f$ on some domain $D$, with zeros having multiplicity at least $k \geq 1$ and such that $f^{n} f^{(k)}$ omits some fixed value $a \neq 0$. Then $\mathscr{F}$ is normal on the domain $D$.

The aim of this paper is to study the zero distribution of the differential polynomial

$$
a(z)(f)^{q_{0}}\left(f^{\prime}\right)^{q_{1}} \ldots\left(f^{(k)}\right)^{q_{k}},
$$

where $a(z)(\not \equiv 0, \infty)$ is a small function of $f$. Moreover, using this value distribution result, we give some normality criterion for family of analytic functions.

2. Main Results. Let $f$ be a transcendental meromorphic function and $a(z)$ be a small function of $f$. Also, let $q_{0}, q_{1}, \ldots, q_{k} \in \mathbb{N} \cup\{0\}$. Let us define

$$
M[f]:=a(z)(f)^{q_{0}}\left(f^{\prime}\right)^{q_{1}} \ldots\left(f^{(k)}\right)^{q_{k}} .
$$

Also, we define $\mu:=q_{0}+q_{1}+\ldots+q_{k}$ and $\mu_{*}:=q_{1}+2 q_{2}+\ldots+k q_{k}$.

Theorem 1. Let $f(z)$ be a transcendental meromorphic function and $\varphi(z)(\not \equiv 0, \infty)$ be a small function of $f(z)$. If $q_{0} \geq 0, q_{k} \geq 1$, then

$$
\mu T(r, f) \leq \bar{N}(r, \infty ; f)+\bar{N}(r, 0 ; f)+\sum_{i=1}^{k} q_{i} N_{i}(r, 0 ; f)+\bar{N}(r, \varphi ; M[f])+S(r, f) .
$$

Remark 1. Clearly Theorem 1 extends Theorem E.

Theorem 2. Let $f(z)$ be a transcendental meromorphic function and $\varphi(z)(\not \equiv 0, \infty)$ be a small function of $f(z)$ such that $\varphi$ and $f$ has no common zero. Moreover, we assume that $\frac{1}{a(z)}$ and $f$ has no common zero. If every pole of $f(z)$ has multiplicity at least $l(\geq 1), q_{0}>1+\frac{1}{l}$ and $q_{k} \geq 1$, then

$$
T(r, f) \leq \frac{1}{q_{0}-1-\frac{1}{l}} N\left(r, \frac{1}{M[f]-\varphi}\right)+S(r, f) .
$$


Corollary 1. Let $f(z)$ be a transcendental entire function and $\varphi(z)(\not \equiv 0, \infty)$ be a small function of $f(z)$ such that $\varphi$ and $f$ has no common zero. Moreover, we assume that $\frac{1}{a(z)}$ and $f$ has no common zero. If $q_{0}>1$ and $q_{k} \geq 1$, then

$$
T(r, f) \leq \frac{1}{q_{0}-1} N\left(r, \frac{1}{M[f]-\varphi}\right)+S(r, f) .
$$

Corollary 2. Let $f(z)$ be a transcendental entire (resp. meromorphic function such that every pole of $f(z)$ has multiplicity at least $l(\geq 1))$ and $\varphi(z)(\not \equiv 0, \infty)$ be a small function of $f(z)$ such that $\varphi$ and $f$ has no common zero. Moreover, we assume that $\frac{1}{a(z)}$ and $f$ has no common zero. If $q_{0}>1$ (resp. $1+\frac{1}{l}$ ) and $q_{k} \geq 1$, then $M[f]-\varphi$ has infinitely many zeros.

Morevoer, as an application of corollary 2, we prove a normality criterion for a family of analytic functions.

Theorem 3. Let $\mathscr{F}$ be a family of analytic functions on a domain $D$ and let $k(\geq 1), q_{0}(\geq 2)$, $q_{i}(\geq 0)(i=1,2, \ldots, k-1), q_{k}(\geq 1)$ be positive integers. If for each $f \in \mathscr{F}: i$. $f$ has only zeros of multiplicity at least $k$, ii. $f^{q_{0}}\left(f^{\prime}\right)^{q_{1}} \ldots\left(f^{(k)}\right)^{q_{k}} \neq 1$, then $\mathscr{F}$ is normal on domain $D$.

\section{Lemmas.}

Lemma 1. For a non-constant meromorphic function $g$, we obtain

$$
N\left(r, \frac{g^{\prime}}{g}\right)-N\left(r, \frac{g}{g^{\prime}}\right)=\bar{N}(r, g)+N\left(r, \frac{1}{g}\right)-N\left(r, \frac{1}{g^{\prime}}\right) .
$$

Proof. The proof is same as the formula (12) of ([5]).

Lemma 2. Let $f$ be a transcendental meromorphic function and $M[f]$ be a differential polynomial defined in (1), then

$$
T(r, M[f])=O(T(r, f)) \text { and } S(r, M[f])=S(r, f) .
$$

Proof. The proof is similar to the proof of the Lemma 2.4 of ([7]).

Lemma 3. Let $f$ be a transcendental meromorphic function and $M[f]$ be a differential polynomial defined in (1) with $q_{0} \geq 1$, then $M[f]$ must be non-constant.

Proof. Here $\left(\frac{1}{f}\right)^{\mu}=a(z)\left(\frac{f^{\prime}}{f}\right)^{q_{1}}\left(\frac{f^{\prime \prime}}{f}\right)^{q_{2}} \ldots\left(\frac{f^{(k)}}{f}\right)^{q_{k}} \frac{1}{M[f]}$. Thus by the first fundamental theorem and lemma of logarithmic derivative, we have

$$
\begin{gathered}
\mu T(r, f) \leq \sum_{i=1}^{k} q_{i} N\left(r, \infty ; \frac{f^{(i)}}{f}\right)+T(r, M[f])+S(r, f) \leq \\
\leq \sum_{i=1}^{k} i q_{i}(\bar{N}(r, 0 ; f)+\bar{N}(r, \infty ; f))+T(r, M[f])+S(r, f) \leq \sum_{i=1}^{k} i q_{i}(N(r, 0 ; M[f])+ \\
+N(r, \infty ; M[f]))+T(r, M[f])+S(r, f) \leq\left(2 \mu_{*}+1\right) T(r, M[f])+S(r, f),
\end{gathered}
$$

Since $f$ is a transcendental meromorphic function, thus $M[f]$ must be non-constant. This completes the proof. 
Lemma 4. ([10]) Let $\mathscr{F}$ be a family of meromorphic functions on the unit disc $\Delta$ such that all zeros of functions in $\mathscr{F}$ have multiplicity at least $k$. Let $\alpha$ be a real number satisfying $0 \leq \alpha<k$. Then $\mathscr{F}$ is not normal in any neighbourhood of $z_{0} \in \Delta$ if and only if there exist (i) points $z_{n} \in \Delta, z_{n} \rightarrow z_{0}$, (ii) positive numbers $\rho_{n}, \rho_{n} \rightarrow 0$ and (iii) functions $f_{n} \in \mathscr{F}$ such that $\rho_{n}^{-\alpha} f_{n}\left(z_{n}+\rho_{n} \zeta\right) \rightarrow g(\zeta)$ spherically uniformly on compact subsets of $\mathbb{C}$, where $g$ is a non-constant meromorphic function.

\section{Proof of the Theorems.}

Proof of Theorem 1. Since $\frac{1}{f^{\mu}}=\frac{M[f]}{f^{\mu}} \frac{1}{M[f]}$, so by the first fundamental theorem and lemma of logarithmic derivative, we have

$$
\begin{gathered}
\mu T(r, f)=N\left(r, \frac{1}{f^{\mu}}\right)+m\left(r, \frac{1}{f^{\mu}}\right)+O(1) \leq N\left(r, 0 ; f^{\mu}\right)+m\left(r, \frac{1}{M[f]}\right)+S(r, f) \leq \\
\leq N\left(r, 0 ; f^{\mu}\right)+T(r, M[f])-N(r, 0 ; M[f])+S(r, f) .
\end{gathered}
$$

Now, by Nevanlinna's three small functions theorem ([2], pp. 47), we have

$$
T(r, M[f]) \leq \bar{N}(r, 0 ; M[f])+\bar{N}(r, \infty ; M[f])+\bar{N}(r, \varphi ; M[f])+S(r, M[f]) .
$$

Let $z_{0}$ be a zero of $f$ with multiplicity $q(\geq 1)$.

Case-I If $q \leq k$, then $z_{0}$ is a zero of $M[f]$ of order at least $q q_{0}+(q-1) q_{1}+(q-2) q_{2}+\ldots+$ $2 q_{q-2}+q_{q-1}+t$ (where $t=0$ if $a(z)$ has no zero or pole at $z_{0} ; t=s$ if $a(z)$ has zero of order $s$ at $z_{0}$, and $t=-s$ if $a(z)$ has pole of order $s$ at $\left.z_{0}\right)$. Now

$$
\begin{gathered}
\mu q+1-\left(q q_{0}+(q-1) q_{1}+(q-2) q_{2}+\ldots+2 q_{q-2}+q_{q-1}\right)-t= \\
=1+\left\{q_{1}+2 q_{2}+\ldots+(q-2) q_{q-2}+(q-1) q_{q-1}\right\}+\left(q q_{q}+q q_{q+1}+\ldots+q q_{k}\right)-t .
\end{gathered}
$$

Case-II If $q \geq k+1$, then $z_{0}$ is a zero of $M[f]$ of order $q \mu-\mu_{*}+t$ (where $t=0$ if $a(z)$ has no zero or pole at $z_{0} ; t=s$ if $a(z)$ has zero of order $s$ at $z_{0}$, and $t=-s$ if $a(z)$ has pole of order $s$ at $z_{0}$ ). Now

$$
\mu q+1-\left(q \mu-\mu_{*}\right)-t=1+q_{1}+2 q_{2}+\ldots+k q_{k}-t .
$$

Thus from the above discussion, we have

$$
N\left(r, 0 ; f^{\mu}\right)+\bar{N}(r, 0 ; M[f])-N(r, 0 ; M[f]) \leq \bar{N}(r, 0 ; f)+\sum_{i=1}^{k} q_{i} N_{i}(r, 0 ; f)+S(r, f) .
$$

Combining (3),(4) and (5), we have

$$
\begin{gathered}
\mu T(r, f) \leq N\left(r, 0 ; f^{\mu}\right)+T(r, M[f])-N(r, 0 ; M[f])+S(r, f) \leq \\
\leq N\left(r, 0 ; f^{\mu}\right)+\bar{N}(r, 0 ; M[f])+\bar{N}(r, \infty ; M[f])+\bar{N}(r, \varphi ; M[f])-N(r, 0 ; M[f])+S(r, f) \leq \\
\leq \bar{N}(r, \infty ; f)+\bar{N}(r, 0 ; f)+\sum_{i=1}^{k} q_{i} N_{i}(r, 0 ; f)+\bar{N}(r, \varphi ; M[f])+S(r, f) .
\end{gathered}
$$

This completes the proof.

Proof of Theorem 2. Let us define $b=b(z)=: \frac{1}{\varphi(z)}$. Now by Lemma 3, it is clear that $b(z) M[f]$ is non-constant. Again

$$
\frac{1}{f^{\mu}}=\frac{b M[f]}{f^{\mu}}-\frac{(b M[f])^{\prime}}{f^{\mu}} \cdot \frac{(b M[f]-1)}{(b M[f])^{\prime}} .
$$


Thus in view of Lemmas 1 and 2, the first fundamental theorem and lemma of logarithmic derivative, we have

$$
\begin{gathered}
\mu m\left(r, \frac{1}{f}\right) \leq m\left(r, \frac{b M[f]}{f^{\mu}}\right)+m\left(r, \frac{(b M[f])^{\prime}}{f^{\mu}}\right)+m\left(r, \frac{b M[f]-1}{(b M[f])^{\prime}}\right)+O(1) \leq \\
\leq 2 m\left(r, \frac{b M[f]}{f^{\mu}}\right)+m\left(r, \frac{(b M[f])^{\prime}}{b M[f]}\right)+m\left(r, \frac{b M[f]-1}{(b M[f])^{\prime}}\right)+O(1) \leq \\
\quad \leq T\left(r, \frac{(b M[f])^{\prime}}{b M[f]-1}\right)-N\left(r, \frac{b M[f]-1}{(b M[f])^{\prime}}\right)+S(r, f) \leq \\
\leq \bar{N}(r, \infty ; f)+N\left(r, \frac{1}{b M[f]-1}\right)-N\left(r, 0 ;(b M[f])^{\prime}\right)+S(r, f) \leq \\
\leq \frac{1}{l} N(r, \infty ; f)+N\left(r, \frac{1}{M[f]-\varphi}\right)-\left(q_{0}-1\right) N(r, 0 ; f)+S(r, f) .
\end{gathered}
$$

Now, using the first fundamental theorem and (7), we obtain

$$
\left(\mu-q_{0}+1\right) m\left(r, \frac{1}{f}\right)+\left(q_{0}-1\right) T(r, f) \leq N\left(r, \frac{1}{M[f]-\varphi(z)}\right)+\frac{1}{l} N(r, \infty ; f)+S(r, f) .
$$

As $q_{0}>1+\frac{1}{l}$, then from (8), we have

This completes the proof.

$$
T(r, f) \leq \frac{1}{q_{0}-1-\frac{1}{l}} N\left(r, \frac{1}{M[f]-\varphi(z)}\right)+S(r, f) .
$$

Proof of Theorem 3. Since normality is a local property, we may assume that $D=\Delta$. If possible, suppose that $\mathscr{F}$ is not normal on $\Delta$, then by Lemma 4 , there exist $\left\{f_{n}\right\} \subset \mathscr{F}$, $z_{n} \in \Delta$ and positive numbers $\rho_{n}$ with $\rho_{n} \rightarrow 0$ such that

$$
g_{n}(\zeta)=\rho_{n}^{-\alpha} f_{n}\left(z_{n}+\rho_{n} \zeta\right) \rightarrow g(\zeta)
$$

locally, uniformly in spherical metric, where we choose $\alpha=\frac{\mu_{*}}{\mu}$. Now, by Lemma $4, g(\zeta)$ is a non-constant meromorphic function, moreover, by Hurwitz's theorem, all zeros of $g(\zeta)$ are of multiplicity at least $k$. Next, we define

$$
H_{n}(\zeta)=\left(g_{n}(\zeta)\right)^{q_{0}}\left(g_{n}^{\prime}(\zeta)\right)^{q_{1}} \ldots\left(g_{n}^{(k)}(\zeta)\right)^{q_{k}}, \quad H(\zeta)=(g(\zeta))^{q_{0}}\left(g^{\prime}(\zeta)\right)^{q_{1}} \ldots\left(g^{(k)}(\zeta)\right)^{q_{k}} .
$$

Thus $H(\zeta) \not \equiv 0$, otherwise, $g(\zeta)$ will become a polynomial of degree at most $k-1$, which is impossible. Also

$$
\begin{gathered}
H_{n}(\zeta)=\rho_{n}^{\mu_{*}-\alpha \mu}\left(f_{n}\left(z_{n}+\rho_{n} \zeta\right)\right)^{q_{0}}\left(f_{n}^{\prime}\left(z_{n}+\rho_{n} \zeta\right)\right)^{q_{1}} \ldots\left(f_{n}^{(k)}\left(z_{n}+\rho_{n} \zeta\right)\right)^{q_{k}}= \\
=\left(f_{n}\left(z_{n}+\rho_{n} \zeta\right)\right)^{q_{0}}\left(f_{n}^{\prime}\left(z_{n}+\rho_{n} \zeta\right)\right)^{q_{1}} \ldots\left(f_{n}^{(k)}\left(z_{n}+\rho_{n} \zeta\right)\right)^{q_{k}} \rightarrow H(\zeta)
\end{gathered}
$$

locally, uniformly in spherical metric. Since, $H_{n}(\zeta) \neq 1$, thus by the Hurwitz's Theorem, $H(\zeta) \neq 1$. Thus by Corollary $2, g(\zeta)$ must be non-constant rational function, otherwise, $H(\zeta)-1$ has infinitely many solution, which is not possible.

Since $\mathscr{F}$ is a family of analytic functions, so $g_{n}(\zeta)$ is analytic. Since, $g_{n}(\zeta) \rightarrow g(\zeta)$ locally, uniformly in spherical metric, so either $g(\zeta) \equiv \infty$, or, $g(\zeta)$ is an analytic function. But, since $g(\zeta)$ is non-constant, so, $g(\zeta)$ must be a polynomial, say, $g(\zeta)=c_{0}+c_{1} \zeta+\ldots+c_{l} \zeta^{l}$. 
If $l \geq k$, then $H(\zeta)$ becomes a non-constant polynomial, which contradicts that $H(\zeta) \neq 1$. Thus $l<k$, which, in view of Hurwitz's Theorem, contradicts our assumptions on zeros of $f \in \mathscr{F}$. Thus our assumption is wrong. So $\mathscr{F}$ is normal. This completes the proof.

Acknowledgement. We are thankful to Prof. C. C. Yang for giving us some ardent help and suggestion in the time of the preparation of this manuscript. We are also grateful to the anonymous referee for his/her valuable suggestions which considerably improved the presentation of the paper.

\section{REFERENCES}

1. H.Y. Chen, M.L. Fang, The value distribution of $f f^{\prime}$, Sci. China Math., 38 (1995), 789-798.

2. W.K. Hayman, Meromorphic functions, The Clarendon Press, Oxford, 1964.

3. W.K. Hayman, Picard values of meromorphic functions and their derivatives, Ann. Math., 70 (1959), 9-42.

4. X. Huang, Y. Gu, On the value distribution of $f^{2} f^{(k)}$, J. Aust. Math. Soc., 78 (2005), 17-26.

5. Y. Jiang, B. Huang, A note on the value distribution of $f^{l}\left(f^{(k)}\right)^{n}$, Arxiv: 1405.3742v1 [math.CV] 15 May 2014.

6. I. Lahiri, S. Dewan, Inequalities arising out of the value distribution of a differential monomial, J. Inequal. Pure Appl. Math., 4 (2003), №2, Article 27.

7. N. Li, L.Z. Yang, Meromorphic function that shares one small functions with its differential polynomial, Kyungpook Math. J., 50 (2010), 447-454.

8. E. Mues, Über ein Problem von Hayman, Math. Z., 164 (1979), 239-259.

9. X.C. Pang, Bloch's principle and normal criterion, Sci. China Ser. A, 33 (1989), 782-791.

10. J. Schiff, Normal families, Springer, New York, 1993.

11. N. Steinmetz, Nevanlinna theory, normal families, and algebraic differential equations, Springer, 2017.

12. J.F. Xu, H.X. Yi, Z.L. Zhang, Some inequalities of differential polynomials, Math. Inequal. Appl., 12 (2009), 99-113.

13. J.F. Xu, H.X. Yi, Z.L. Zhang, Some inequalities of differential polynomials II, Math. Inequal. Appl., 14 (2011), 93-100.

14. J.F. Xu, H.X. Yi, A precise inequality of differential polynomials related to small functions, Journal of mathematical inequalities, 10 (4) (2016), 971-976.

15. J. Xu, S. Ye, On the zeros of the differential polynomial $\varphi(z) f(z)^{2}\left(f^{\prime}(z)\right)^{2}-1$, Mathematics, doi:10.3390/math7010087.

16. Q.D. Zhang, A growth theorem for meromorphic functions, J. Chengdu Inst. Meteor., 20 (1992), 12-20.

Department of Mathematics

Ramakrishna Mission Vivekananda Centenary College

Rahara, West Bengal, India,

bikashchakraborty.math@yahoo.com

Department of Mathematics

China University of Petroleum

Qingdao, P.R. China

luwr@upc.edu.cn 